\title{
Estimating joint space of the knee during weight-bearing squatting activity using motion capture - Preliminary results of a new method
}

\author{
J. Clément ${ }^{\mathrm{a} *}$, T. Cresson ${ }^{\mathrm{a}}$, N. Hagemeister ${ }^{\mathrm{a}}$, R. Dumas ${ }^{\mathrm{b}}$, J.A. de Guise ${ }^{\mathrm{a}}$ \\ ${ }^{\mathrm{a}} \mathrm{LIO}$, CRCHUM, École de technologie supérieure, Montréal, Canada \\ bIFSTTAR, LBMC, UMR_T9406, Université Lyon 1, Lyon, France
}

Keywords: joint space; articular contact kinematics; motion capture; optimization; squatting activity

\section{Introduction}

Over the last 10 years, several studies analyzed 3D joint space of the knee and deduced articular contact kinematics during various weight-bearing activities $(\mathrm{Li}$ et al. 2015). Joint space and articular contact kinematics provide relevant data on how the intrinsic biomechanics of the knee is altered after a disease or a surgical procedure, such as knee osteoarthritis (OA) or total knee arthroplasty ( $\mathrm{Li}$ et al. 2015).

Although very accurate, the methods employed by these studies require complex acquisition protocols, implying 3D/2D registration techniques or MRI-based methods, which turn out unusable in clinical routine. The goal of the present study is to assess the feasibility of a new method to estimate 3D joint space of the knee during dynamic weight-bearing squats, which are the $2^{\text {nd }}$ most studied activity in orthopedics (Fukagawa et al. 2012), performed by healthy and OA subjects. To our knowledge, this method based on motion capture has never been proposed before.

\section{Methods}

\subsection{Subjects}

This preliminary study involves two subjects: one healthy woman (60 years, $58.3 \mathrm{~kg}, 156 \mathrm{~cm}$ ) and one OA woman (64 years, $77.8 \mathrm{~kg}, 150 \mathrm{~cm}$ ).

\subsection{Kinematic acquisition and optimization}

3D knee kinematics was recorded on the two subjects while performing dynamic weight-bearing squats $\left(0^{\circ}\right.$ $60^{\circ}-0^{\circ}$ of knee flexion-extension during 10 seconds) with the KneeKG ${ }^{\mathrm{TM}}$ (Emovi Inc., Laval, QC, Canada) and Polaris Spectra ${ }^{\circledR}$ camera $(60-\mathrm{Hz}$, NDI, Waterloo, $\mathrm{ON}$, Canada). The dynamic squats were standardized with a proprioceptive jig (Clément et al. 2014). The $\mathrm{KneeKG}^{\mathrm{TM}}$ provides repeatable and reliable measurements (Lustig et al. 2012), but they are still influenced by soft tissue artefacts (STA) with kinematics errors up to $7^{\circ}$ and $11 \mathrm{~mm}$ (Südhoff et al. 2007). These STA errors were therefore corrected with a multi-body optimization method (MBO) (Gasparutto et al. 2015)

The lower limb model used in MBO consisted of 4 segments (pelvis, femur, tibia, foot) imposing spherical joint constraints at the hip and ankle, and parallel mechanism constraints at the knee. The parallel mechanism was composed of 2 sphere-onplane contacts and 4 deformable ligaments and was personalized from the subject-specific 3D knee bone models. These 3D models were obtained from the upright low-dose biplane radiographic imaging EOS $^{\circledR}$ system (EOS Imaging Inc., Paris, France) with an accuracy around $2 \mathrm{~mm}$ (Cresson et al. 2010).

\subsection{Knee joint space calculation}

Fusion of the 3D bones models of subjects with the optimized knee kinematics allowed computing the 3D joint space during a descent phase of the recorded squats. We established a distance map between the two meshes of the femur and tibia at each instant of the movement, according to the symmetric Hausdorff distance definition (Aspert et al. 2002).

\subsection{Validation}

The validation process was done by comparing the distance maps obtained using our method with those obtained during a quasi-static squat recorded with the EOS $^{\circledR}$ system. The quasi-static squat consisted of 4 positions of knee flexion and was standardized with the same proprioceptive jig used during the dynamic squat. The method used to obtain the 3D knee models for these 4 positions is detailed in Kanhonou et al. (2014) and presents an accuracy and a precision about $0.3^{\circ}$ and $0.3 \mathrm{~mm}$ in terms of positions and orientations of knee bones. Calculation of distance maps was the same as detailed above.

\section{Results and discussion}

The two figures below show the distance maps of the healthy and OA subjects obtained from the recordings of the $\mathrm{KneeKG}^{\mathrm{TM}}$ and $\mathrm{EOS}^{\circledR}$ systems. 


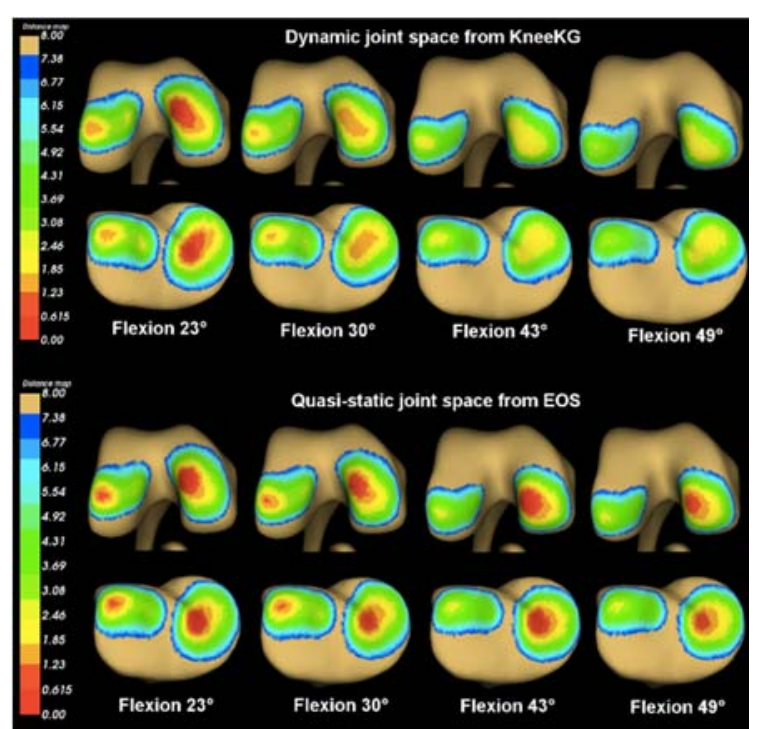

Figure 1 Knee distance maps of a healthy subject

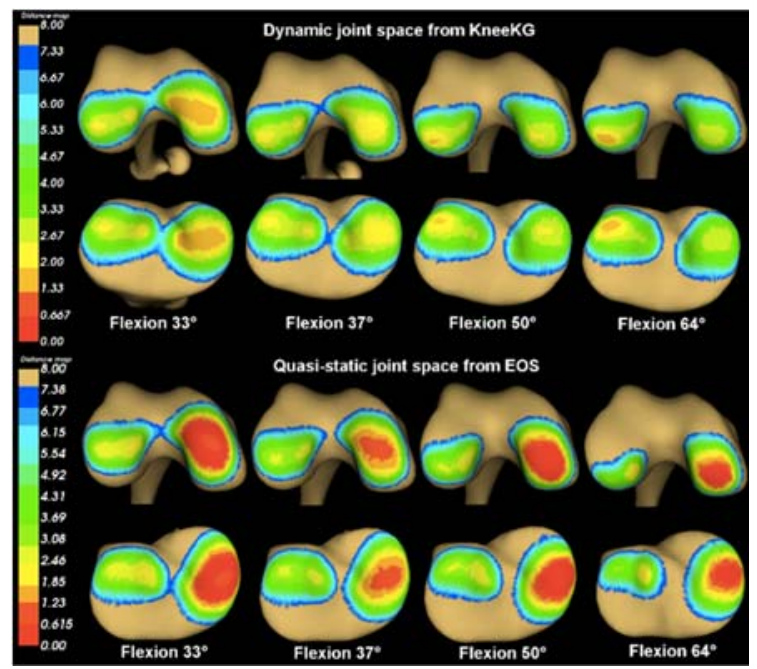

Figure 2 Knee distance maps of an OA subject

On average, measurements based on the KneeKG ${ }^{\mathrm{TM}}$ recordings overestimates of about $2 \mathrm{~mm}$ the distance between the articular surfaces of the femur and tibia compared to the measurements based on the $\operatorname{EOS}^{\circledR}$ system. However, the patterns of the distance maps and the positions of the points of closest contact remain qualitatively similar between the two methods. This could be very interesting to assess the joint reaction forces and torques in the context of a musculoskeletal modeling of the lower limb for example.

\section{Conclusions}

The estimation of joint space using a new method based on motion capture showed very encouraging results. These results nevertheless remain very preliminary. Further investigations on a greater number of subjects, i.e. 10 healthy and $10 \mathrm{OA}$, and exhibiting more quantitative data, i.e. the area covered by the distance maps on the bones and the position of the points of closest contact between the bones, are ongoing.

\section{Acknowledgements}

This work was funded in part by the FRQNT, FRQ-S, NSERC, the CIHR MENTOR program, and grant from Region Rhone-Alpes.

\section{References}

Aspert N, Santa-Cruz D, Ebrahimi T. MESH: measuring errors between surfaces using the Hausdorff distance. Proceedings of the IEEE ICME; 2002.

Clément J, Hagemeister N, Aissaoui R, de Guise JA. 2014. Comparison of quasi-static and dynamic squats: A three-dimensional kinematic, kinetic and electromyographic study of the lower limbs. Gait Posture. 40:94-100.

Cresson T, Branchaud D, Chav R, Godbout B, de Guise JA. 3D shape reconstruction of bone from two $\mathrm{x}$-ray images using $2 \mathrm{D} / 3 \mathrm{D}$ non-rigid registration based on moving least-squares deformation. Proceedings of the SPIE: Image Processing; 2010.

Fukagawa S, Leardini A, Callewaert B, Wong PD, Labey L, Desloovere K, Matsuda S, Bellemans J. 2012. Age-related changes in kinematics of the knee joint during deep squat. The Knee. 19:208212.

Gasparutto X, Sancisi N, Jacquelin E, Parenti-Castelli V, Dumas R. 2015. Validation of a multi-body optimization with knee kinematic models including ligament constraints. J Biomech. 48:1141-1146.

Kanhonou M, Cresson T, Lavoie F, Clément J, Hagemeister N, de Guise JA. 2014. A method to study $3 \mathrm{D}$ knee pseudo-kinematics using low-dose stereoradiography during static squat. Comput Methods Biomech Biomed Eng. 17:138-139.

Li C, Hosseini A, Tsai T-Y, Kwon Y-M, Li G. 2015. Articular contact kinematics of the knee before and after a cruciate retaining total knee arthroplasty. J Orth Res. 33:349-358.

Lustig S, Magnussen R, Cheze L, Neyret P. 2012. The KneeKG system: a review of the literature. Knee Surgery, Sports Traumatology, Arthroscopy. 2012/04/01; 20:633-638.

Südhoff I, Van Driessche S, Laporte S, de Guise JA, Skalli W. 2007. Comparing three attachment systems used to determine knee kinematics during gait. Gait Posture. 25:533-543. 